\title{
Dark Currents in a Fully-Depleted LWIR HgCdTe $P$-on- $n$ Heterojunction: Analytical and Numerical Simulations
}

\author{
J. SCHUSTER ${ }^{10},{ }^{1,2,4}$ R.E. DEWAMES, ${ }^{3}$ and P.S. WIJEWARNASURIYA ${ }^{1}$ \\ 1.-U.S. Army Research Laboratory, 2800 Powder Mill Road, Adelphi, MD 20783, USA. \\ 2.-Department of Electrical and Computer Engineering, Boston University, 8 St. Marys Street, \\ Boston, MA 02215, USA. 3.-Manufacturing Techniques, MTEQ, 10440 Furnace Road, Suite 204, \\ Lorton, VA 22079, USA. 4.-e-mail: jonathan.schuster2.civ@mail.mil
}

In this paper, we use the theory of Evans and Landsberg, which is a generalization of the Shockley-Read-Hall recombination statistics in the space charge region (SCR), to include effects of Auger and radiative recombination processes that are also of origin in the SCR. Using analytical expressions for the current density, we calculate the total dark current density for a variety of conditions. Contributions include radiative and Auger transitions of origin in both the quasi-neutral region and the SCR. Numerical simulations are used to assess the nature of the limitations associated with the analytical calculation in the $n$-extrinsic region $\left(N_{\mathrm{d}} \gg n_{\mathrm{i}}\right.$, where $N_{\mathrm{d}}$ is the doping concentration and $n_{\mathrm{i}}$ is the intrinsic carrier concentration), and to extend the calculations to operating temperatures in the intrinsic region $\left(n_{\mathrm{i}} \gg N_{\mathrm{d}}\right)$. Major findings include the observation that in a fully depleted $P^{+} n$ doublelayer planar hetero-structure, at a reverse bias voltage sufficiently high to suppress the Auger process, SRH centers are not limiting, and the dark current is due to radiative transitions of origin in the $n$-side SCR. From the numerical simulations, while the Auger recombination rate changes drastically with varying the carrier concentration (such as applying reverse bias), the radiative recombination rate remains nearly invariant to varying the carrier concentration, and, as such, does not appreciably change with increasing reverse bias. Using the theory of van Roosbroeck and Shockley, the radiative recombination rate is obtained by integrating the measured optical absorption coefficient over all photon energies. Hence, the theory links the measured absorption coefficient to the measured dark current density for conditions in which the dominant current component is due to radiative recombination. Finally, the numerical simulations reveal, in both the $n$-extrinsic and intrinsic operating regions, that, under sufficient conditions, the detector is radiatively limited.

Key words: $\mathrm{HgCdTe}$, infrared detectors, depleted $p-n$ junction, radiative recombination, Auger suppression, numerical simulations

\section{INTRODUCTION}

Depleted photovoltaic devices operate under the situation that the space charge region (SCR) occupies a significantly larger volume of the active layer

(Received February 27, 2017; accepted August 4, 2017; published online August 21, 2017) than the quasi-neutral region (QNR). Expected is a corresponding reduction in Auger (e-e) transitions, in the $n$-extrinsic region of operation $\left(N_{\mathrm{d}} \gg n_{\mathrm{i}}\right)$, where $N_{\mathrm{d}}$ is the doping concentration and $n_{\mathrm{i}}$ is the intrinsic carrier concentration. In fact, fully depleted devices have no QNR region and instead the entire active layer is depleted. Germane to the understanding of the fully depleted junction characteristics are the analytical expressions for the 
current density $J(V, T)$ derived by Evans and Landsberg. ${ }^{1}$ They provide a generalization of the SRH recombination statistics in the SCR to include effects of Auger and radiative transitions also of origin in the SCR. The current density through a $p-$ $n$ junction is then a sum of recombination currents in both the SCR and the QNR. The authors only considered the case of moderate bias voltage, subject to conditions where the mass action laws are favorable. In this work, numerical methods are used to determine the solutions of Poisson's equation and the electron and hole continuity equations to extend the calculations to include the intrinsic region of operation $\left(n_{\mathrm{i}} \gg N_{\mathrm{d}}\right)$. Given the large SCR present in depleted devices, and very small QNR, the recombination statistics for Auger and radiative effects are vital to properly model the dark current and cannot be neglected.

To calculate the radiative recombination rate, we use the theory of van Roosbroeck and Shockley ${ }^{2}$ where the measured optical absorption coefficient is integrated over all photon energies to yield the radiative generation rate $\left(G_{R}\right)$. This approach is supported by its prior use to explain the observed diffusion current in short-wavelength infrared (IR) heterojunction photodiodes in large format dense focal plane arrays, such as $P^{+} n \operatorname{In}_{0.53} \mathrm{Ga}_{0.47} \mathrm{As} / \mathrm{InP}{ }^{3}$ In this case, the Auger and SRH generation-recombination $(G-R)$ components are about two orders of magnitude smaller than the radiative component calculated by the van Roosbroeck and Shockley theory.

The analytical and numerical methods for analysis of real devices described in this paper enable the description of the dominant dark current density in the QNR and SCR for conditions that include the fully depleted $P^{+} n$ double-layer planar hetero-structure (DLPH) in both the $n$-extrinsic $\left(N_{\mathrm{d}} \gg n_{\mathrm{i}}\right)$ and intrinsic $\left(n_{\mathrm{i}} \gg N_{\mathrm{d}}\right)$ regions of detector operation. The device architecture studied in this paper is a regular DLPH subject to the requirement that the absorbing layer is fully depleted; the larger gap $P^{+}$ layer essentially provides an $n$-side shallow step junction.

Important to the study and results reported here is the recently reported capability, described by Lee et al., ${ }^{4}$ to significantly reduce the indium doping level from the usual doping level of $10^{15} \mathrm{~cm}^{-3}$ to the range of $(2-5) \times 10^{13} \mathrm{~cm}^{-3}$ in $n$-type $\mathrm{HgCdTe}$ materials grown by molecular beam epitaxy (MBE). Lower doping levels will enable larger absorbing layers to be fully depleted at modest reverse bias, hence high quantum efficiency (QE). Expected is a corresponding reduction in Auger (ee) transitions, in the $n$-extrinsic region of operation $\left(N_{\mathrm{d}} \gg n_{\mathrm{i}}\right)$, due to the lower carrier concentration. Therefore, the physics of the SCR are vital in understanding the device operation and in developing optimum designs in terms of junction formation, doping levels and band gap engineering. Essential to being limited by radiative recombination is the need for the Shockley-Read-Hall (SRH) lifetimes to be sufficiently long to ensure that the $G-R$ current due to SRH centers in the SCR will not be the dominant current. Fortunately, the SRH lifetimes are expected to be very long in state-of-the-art $\mathrm{HgCdTe},{ }^{5,6}$ and hopefully the SRH $G-R$ current of origin in the SCR can be mitigated in real devices needed for a variety of applications.

The remainder of the manuscript is organized as follows: "Device Geometry: Double-Layer Planar Hetero-structure" section presents the device geometry, "Analytical Dark Current Model" section derives the analytical dark current model using the generalized Evans and Landsberg current density equations, "Conventional LWIR Detector" section presents analytically modeled results for a conventional LWIR detector, "Depleted LWIR Detector" section presents analytically modeled results for a depleted LWIR detector, "Numerical Validation of Analytical Calculation and Assumptions" section presents numerically simulated results that corroborate the analytical results while discussing the assumptions used in the analytical calculation, "Intrinsic Region" section utilizes numerical simulations to extend the Evans and Landsberg theory from the $n$-extrinsic to the intrinsic region, and finally "Conclusions" offers some concluding remarks. Additionally, "Appendix 1" outlines the generalized Evans and Landsberg current density equations with pertinent derivations and "Appendix 2" presents the numerical approach used in the "Numerical Validation of Analytical Calculation and Assumptions" and "Intrinsic Region" sections.

\section{DEVICE GEOMETRY: DOUBLE LAYER PLA- NAR HETERO-STRUCTURE}

The photodiode studied to exploit the physical underlying properties of depleted $p-n$ junctions is a DLPH $P^{+} n \mathrm{Hg}_{1-x} \mathrm{Cd}_{x}$ Te photodiode. ${ }^{7,8}$ The device is composed of a narrow-bandgap $n$-type absorber layer (AL) and a wide-bandgap $n$-type cap layer (CL) in a planar configuration. The layers are grown by MBE on CdZnTe substrates with an intermediate wide-bandgap buffer layer (BL). A schematic of the device architecture is shown in Fig. 1 and the parameters of the electrically active layers are given in Table I. The junction is formed by ion implantation of arsenic to form a $p$-type region. The penetration depth of the arsenic $\left(z_{j}\right.$, shown in Fig. 1, notation formulated by Grimbergen ${ }^{9}$ ) can be controlled to yield either shallow $\left(z_{j} \geq 0 \mu \mathrm{m}\right)$ or deep $\left(z_{j}<0 \mu \mathrm{m}\right)$ junctions. The term "hetero-structure" is used to denote the situation where the transition from $P^{+}$to $n$ is situated at the metallurgical interface $\left(z_{j}=0 \mu \mathrm{m}\right)$, under these conditions the photodiode is basically an abrupt step-junction with the $n$-side SCR and QNR positioned in the narrow- 
bandgap AL. "Heterojunction" is used to denote the situation where the transition from $P^{+}$to $N$ is positioned into the wide-bandgap CL $\left(z_{j}<0 \mu \mathrm{m}\right)$. The latter photodiode design provides for suppression of

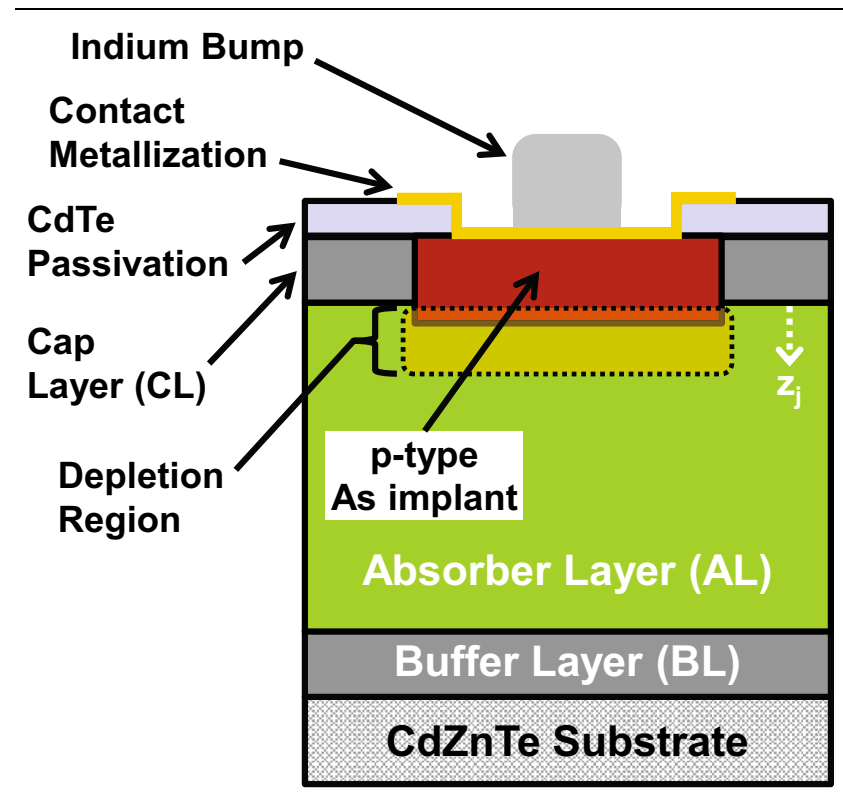

Fig. 1. DLPH ion implanted $P^{+} n \mathrm{Hg}_{1-x} \mathrm{Cd}_{x}$ Te photodiode architecture. ${ }^{7,8}$ For illustration, the vector $z_{j}(0 \mu \mathrm{m})$ is also indicated (not to scale). non-ideal dark current components generated by $G-R$ SRH transitions ${ }^{10,11}$ and/or trap-assisted tunneling (TAT). For simplicity, in this work, we set $z_{j}=0.0 \mu \mathrm{m}$.

\section{ANALYTICAL DARK CURRENT MODEL}

It is now instructive to consider the analytical dark current in the $n$-extrinsic region $\left(N_{\mathrm{d}} \gg n_{\mathrm{i}}\right)$ of the $P^{+} n$ DLPH $\left(z_{j}=0 \mu \mathrm{m}\right)$ derived from the Evans and Landsberg equations, ${ }^{1}$ under the condition $V_{j}<-2 / \beta_{T}$, where $V_{j}$ is the applied bias voltage, $\beta_{T}=1 / k_{B} T, k_{B} T$ is the thermal voltage, $k_{B}$ is the Boltzmann constant and $T$ is the diode temperature. The total reverse bias dark current density $\left(J_{\mathrm{d}-\text { total }}=J_{\mathrm{d} \text {-diff. }}+J_{G-R \text {-unav.-rad }}+J_{G-R \text {-unav.-Aug }}\right)$, under the above condition, is given by

$$
\begin{gathered}
J_{\mathrm{d}-\text { total }}\left(V_{j}, T\right)=-q n_{\mathrm{i}}^{2}\left\{\left(B_{0}+n_{n 0} B_{1}\right)\left[d_{n}-W_{\text {dep }, \mathrm{n}-\text { side }}\left(V_{j}\right)\right]\right. \\
\left.+B_{0} W_{\text {dep }, n-\text { side }}\left(V_{j}\right)+k_{B} T\left(\frac{n_{n 0} B_{1}+p_{p 0} B_{2}}{E\left(V_{j}\right)}\right)\right\}
\end{gathered}
$$

where $q$ is the electronic charge, $V_{b i}$ is the built-in voltage, $B_{0}$ is the radiative recombination coefficient, $B_{1}$ is the Auger- 1 (e-e) recombination coefficient, $B_{2}$ is the Auger-7 (h-h) recombination coefficient, $n_{\mathrm{i}}$ is the intrinsic carrier concentration, $n_{n 0}$ is the majority carrier density on the $n$-side, $p_{p 0}$

\begin{tabular}{|c|c|c|c|c|c|c|}
\hline \multirow[b]{2}{*}{ Parameter } & \multirow[b]{2}{*}{ Unit } & \multirow{2}{*}{$\frac{\text { Cap }}{\boldsymbol{P}^{+}}$} & \multicolumn{4}{|c|}{ Absorber } \\
\hline & & & $\boldsymbol{n}$ & & $n^{-}$ & \\
\hline Temperature & $\mathrm{K}$ & 60 & 60 & 60 & 125 & 200 \\
\hline Mole fraction $(x)$ & & 0.2750 & 0.2133 & 0.2133 & 0.2133 & 0.2133 \\
\hline Dielectric constant $(\epsilon)$ & $\epsilon_{0}$ & 16.67 & 17.45 & 17.45 & 17.45 & 17.45 \\
\hline Dielectric constant $\left(\epsilon_{\infty}\right)$ & $\epsilon_{0}$ & 11.92 & 12.57 & 12.57 & 12.57 & 12.57 \\
\hline$E_{g}$ & $\mathrm{eV}$ & 0.1992 & 0.0993 & 0.0993 & 0.1192 & 0.1422 \\
\hline Gap wavelength $\left(\lambda_{g}\right)$ & $\mu \mathrm{m}$ & 6.22 & 12.49 & 12.49 & 10.40 & 8.72 \\
\hline Cutoff wavelength $\left(\lambda_{c o}\right)$ & $\mu \mathrm{m}$ & & 12.60 & 12.15 & 9.89 & 8.14 \\
\hline Thickness $\left(d_{n}\right)$ & $\mu \mathrm{m}$ & 0.1 & 12.0 & 2.0 & 2.0 & 2.0 \\
\hline$n_{\mathrm{i}}$ & $\mathrm{cm}^{-3}$ & $2.742 \times 10^{8}$ & $2.698 \times 10^{12}$ & $2.698 \times 10^{12}$ & $5.544 \times 10^{14}$ & $5.352 \times 10^{15}$ \\
\hline Electrical type & & $p$ & $n$ & $n$ & $n$ & $n$ \\
\hline Doping $\left(N_{D A}\right)$ & $\mathrm{cm}^{-3}$ & $5.697 \times 10^{16}$ & $1.000 \times 10^{15}$ & $5.000 \times 10^{14}$ & $5.000 \times 10^{14}$ & $5.000 \times 10^{14}$ \\
\hline Built in voltage $\left(V_{b i}\right)$ & $\mathrm{V}$ & & 0.08207 & 0.07848 & 0.05466 & 0.04182 \\
\hline$F_{1} F_{2}$ & & & 0.2 & 0.2 & 0.2 & 0.2 \\
\hline$B_{0}$ & $\mathrm{~cm}^{3} \mathrm{~s}^{-1}$ & & $9.924 \times 10^{-11}$ & $9.924 \times 10^{-11}$ & $4.453 \times 10^{-11}$ & $2.882 \times 10^{-11}$ \\
\hline$B_{1}$ & $\mathrm{~cm}^{6} \mathrm{~s}^{-1}$ & & $1.355 \times 10^{-24}$ & $1.355 \times 10^{-24}$ & $3.242 \times 10^{-25}$ & $1.086 \times 10^{-25}$ \\
\hline$B_{2}$ & $\mathrm{~cm}^{6} \mathrm{~s}^{-1}$ & & $2.227 \times 10^{-25}$ & $2.227 \times 10^{-25}$ & $5.267 \times 10^{-26}$ & $1.746 \times 10^{-26}$ \\
\hline$\tau_{\mathrm{rad}}$ & $\mu \mathrm{s}$ & & 10.08 & 20.15 & 18.46 & 3.238 \\
\hline$\tau_{\text {Auger-1 }}$ & $\mu \mathrm{s}$ & & 0.738 & 2.952 & 2.767 & 0.134 \\
\hline$\gamma_{\text {Auger-7 }}$ & & & 6.085 & 6.085 & 6.157 & 6.222 \\
\hline$\tau_{\text {net }}$ & $\mu \mathrm{s}$ & & 0.688 & 2.575 & 2.406 & 0.128 \\
\hline
\end{tabular}

Table I. $\mathrm{Hg}_{1-\mathrm{x}} \mathrm{Cd}_{\mathrm{x}} \mathrm{Te}$ material and layer parameters used in both the analytical and numerical simulations

Shown is the CL and AL at $\mathrm{T}=60 \mathrm{~K}$ and a lower doped AL at $\mathrm{T}=60 \mathrm{~K}\left(\mathrm{~N}_{\mathrm{d}} \gg \mathrm{n}_{\mathrm{i}}\right), \mathrm{T}=125 \mathrm{~K}\left(\mathrm{n}_{\mathrm{i}} \sim \mathrm{N}_{\mathrm{d}}\right)$, and $\mathrm{T}=200 \mathrm{~K}\left(\mathrm{n}_{\mathrm{i}} \gg \mathrm{N}_{\mathrm{d}}\right)$. 
is the majority carrier density on the $p$-side, $d_{n}$ is the $n$-side device thickness, $W_{\text {dep,n-side }}\left(V_{j}\right)$ is the $n$ side width of the SCR, and

$$
E\left(V_{j}\right)=\frac{V_{b i}-V_{j}}{W_{\text {dep }, \text { n-side }}\left(V_{j}\right)},
$$

where $E\left(V_{j}\right)$ is $1 / 2$ the maximum electric field of an abrupt one-sided step junction in the depletion approximation. For the details of the derivation of Eqs. 1 and 2 from the original Evans and Landsberg equations, see "Appendix 1". Note that, since contributions from the $P^{+}$-side are negligible in the $P^{+} n$ one-sided step junction, those contributions have been omitted here in the expression for the diffusion current. Equation 1 is the sum of three terms: radiative and Auger transitions in the $n$-side $\mathrm{AL}$ QNR $\left(J_{\mathrm{d} \text {-diff. }}\right)$, radiative transitions in the AL SCR region $\left(J_{G-R \text {-unav.-rad }}\right)$, and Auger transitions in the AL SCR region $\left(J_{G-R-\text { unav--Aug }}\right)$ respectively. When the device is fully depleted, $W_{\text {dep,n-side }}=d_{n}$, causing $J_{\text {d-diff. }} \rightarrow 0$ and Eq. 1 reduces to

$$
J_{\mathrm{d}-\text { total }}\left(V_{j}, T\right)=-q n_{\mathrm{i}}^{2}\left\{B_{0} d_{n}+k_{B} T \frac{n_{n 0} B_{1}+p_{p 0} B_{2}}{E^{\prime}\left(V_{j}\right)}\right\},
$$

where

$$
E^{\prime}\left(V_{j}\right)=\frac{V_{b i}-V_{j}}{d_{n}} .
$$

Note that the Auger terms are divided by the electric field $E^{\prime}\left(V_{j}\right)$, whereas the $B_{0}$ radiative term is not. As such, the Auger component $J_{G-R \text {-unav.-Aug }}$ can be avoided, since it is divided by the electric

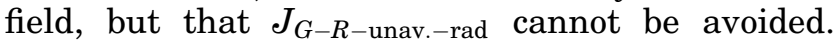
Since $E^{\prime}\left(V_{j}\right)$ increases linearly with reverse bias for a fully depleted diode, eventually, the Auger term vanishes and the radiative term will dominate. To be limited by radiative transitions when the diode is fully depleted, the condition is

$$
B_{0}>\left(n_{n o} B_{1}+p_{p 0} B_{2}\right) \frac{k_{B} T}{V_{b i}-V_{j}} .
$$

This clearly shows that the large applied reverse voltage asymptotic limiting dark current, such that the diode is fully depleted, is expected to be

$$
J_{\text {Fully-Depleted }}=-q G_{R} d_{n},
$$

where $G_{R}\left(=n_{\mathrm{i}}^{2} B_{0}\right)$ is the radiative recombination rate in the $\mathrm{AL}$, equal to integrating the absorption coefficient over all photon energies. ${ }^{12}$ Note that the theory of Evans and Landsberg did not exploit the large reverse bias characteristics and instead focused on forward bias. Their results have been extended here for reverse bias. For the remainder of the details, see "Appendix 1".

Therefore, upon extending the Evans and Landsberg theory, which includes in the SCR the sum of intrinsic direct radiative and Auger transitions, the extended expressions predict that the asymptotic high reverse bias dark current in a fully depleted diode is the product of the radiative recombination rate and the absorber thickness with no Auger dependence, applicable to any wavelength region. In this model, the limiting dark current component is radiative, hence the maximum high temperature for background limited performance (BLIP) operation and for size, weight, power and cost (SWAP-C) reductions is determined by

$$
\frac{J(V, T)}{J_{\text {photon }}}=\frac{G_{R} d_{n}}{\eta \phi}<1
$$

where $\eta$ is the $\mathrm{QE}$ and $\phi$ the photon flux. This enables the prospect of engineering LWIR detectors that eliminate Auger recombination, such that they are radiatively limited, with performance advantages in the $n$-extrinsic and intrinsic regions of operations including potential SWAP-C benefits associated with high-operating-temperature (HOT) detectors.

\section{CONVENTIONAL LWIR DETECTOR}

It is useful as a reference to calculate analytically

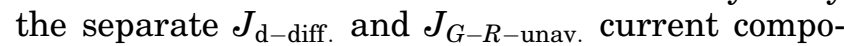
nents from the Evans and Landsberg theory given by Eq. 1 for a conventional LWIR $P^{+} n$ DLPH hetero-structure design. The structure is designed to have a high QE with AL thickness $d_{n}=12 \mu \mathrm{m}$ and doping $N_{\mathrm{D}}=1 \times 10^{15} \mathrm{~cm}^{-3}$. The dark current density as a function of 1000 times inverse temperature for a bias voltage $V_{j}=-0.05 \mathrm{~V}$ is shown in Fig. 2. For these conditions, assuming no $\mathrm{SRH}$ transitions via band-gap states, the dominant contribution to the dark current is from carrier diffusion of origin in the $n$-side QNR of the junction. Also shown in Fig. 2 is the empirical "Rule 07" equation, which is a fit to DLPH detectors built by Teledyne Imaging Sensors. ${ }^{13}$ These detectors are built for high QE, hence the thickness of the absorbing layer $d_{n} \sim$ cut-off wavelength $\left(\lambda_{c o}\right) \sim \lambda_{g}$. The empirical Rule 07 equation, valid for $\mathrm{Hg}_{1-x} \mathrm{Cd}_{x} \mathrm{Te}$ with $x \leq 0.3$, is given by

$$
J=J_{0} \exp \left[C \frac{1.24}{\lambda_{c o} k_{B} T}\right]
$$

where $C=-1.16239$ and $J_{0}=8367 \mathrm{~A} / \mathrm{cm}^{2}$. For the condition that $\lambda_{c o} \approx \lambda_{g}$, Eq. 8 becomes

$$
J=J_{0} \exp \left[C \frac{E_{g}(x, T)}{k_{B} T}\right]
$$

where the band-gap energy $E_{g}(x, T)=1.24 / \lambda_{g}(x, T)$. For $\mathrm{Hg}_{1-x} \mathrm{Cd}_{x} \mathrm{Te},{ }^{14} E_{g}(x, T)=E_{g}(x, 0)+\alpha(1-2 x) T$, where $\alpha=5.35 \times 10^{-4} \mathrm{eV} / \mathrm{K}$. Inserting the equation for $E_{g}(x, T)$ into Eq. 9, we obtain

$$
J=J_{0} \exp \left[C(1-2 x) \frac{\alpha}{k}\right] \exp \left[C \frac{E_{g}(x, 0)}{k_{B} T}\right]
$$

where $E_{g}(x, 0)$ in the exponent of the Arrhenius equation is the energy gap at $T=0 \mathrm{~K}$, given by 


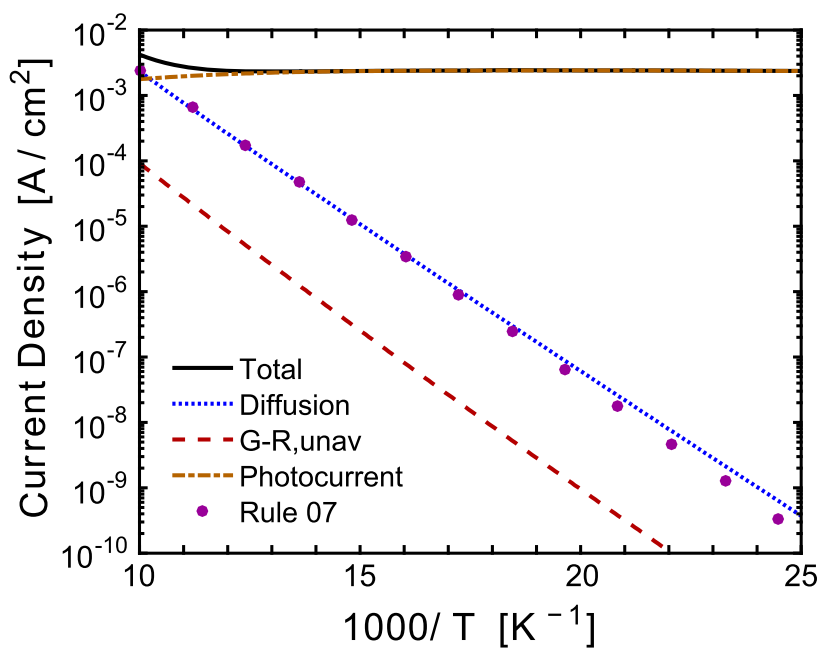

Fig. 2. Analytically modeled LWIR $\mathrm{HgCdTe} P^{+} n$ dark current density versus 1000 times inverse temperature at an applied bias of $V_{j}=-0.05 \mathrm{~V}$. The material parameters used are given in Table I (columns $n$ and $P^{+}$). Also plotted is the photocurrent from a $300-\mathrm{K}$ blackbody, with F/3 optics, over the 8- to $12-\mu \mathrm{m}$ spectral band and assuming no reflection losses. The Rule $07^{13}$ dark current is also plotted for comparison.

$$
E g(x, 0)=-0.302+1.93 x-0.81 x^{2}+0.832 x^{3} .
$$

The fitting parameter $C$ is negative and greater than unity, hence deviation from the ideal diode $C=-1$, is expected at low temperature as seen by comparing the Arrhenius plot for the diffusion current with the empirical Rule 07 calculation for $x=0.2133, E_{g}(0.2133,0)=8.0892 \times 10^{-2} \mathrm{eV}$. Equation 10 is a convenient expression for using Rule 07 , for comparative purposes, keeping in mind the underlying design parameters such as $d_{n}=12 \mu \mathrm{m}$, $\lambda_{c 0}=12.6 \mu \mathrm{m}$, and $N_{\mathrm{D}} \sim 10^{15} \mathrm{~cm}^{-3}$.

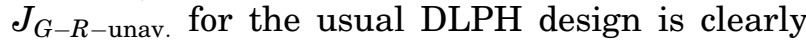
significantly smaller than $J_{\text {d-diff. }}$ and can be neglected. The calculated dark current assumed only the $n$-side contributed to the diffusion current; the agreement with the Rule 07 prediction supports that LWIR conventional DLPH detectors are $n$ sided step junction hetero-structures, limited by Auger (e-e) recombination. As shown in Table I ( $n$ column), the device is clearly Auger limited $\left(\tau_{\text {Auger }-1} \ll \tau_{\text {rad }}\right)$. From the above calculation and comparisons with the empirical Rule 07 results,

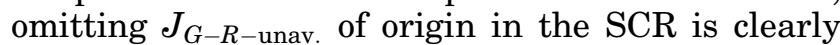
justified in the reference structure.

\section{DEPLETED LWIR DETECTOR}

With the advent of the capability to significantly reduce $N_{\mathrm{D}}$, to values in the low-to-mid $10^{13} \mathrm{~cm}^{-3}$ range, ${ }^{4}$ clearly expected in the $n$-extrinsic region is the possibility to significantly reduce Auger

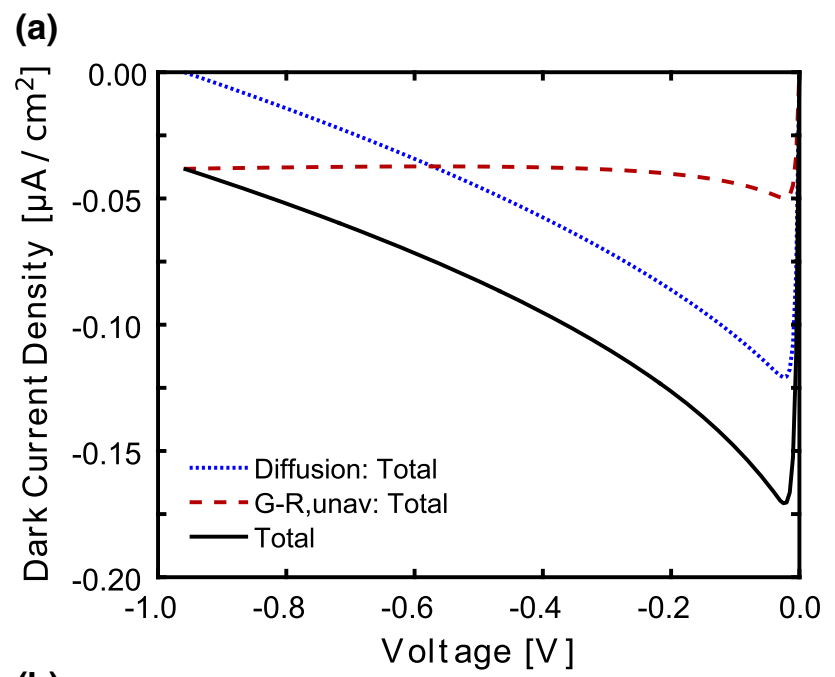

(b)

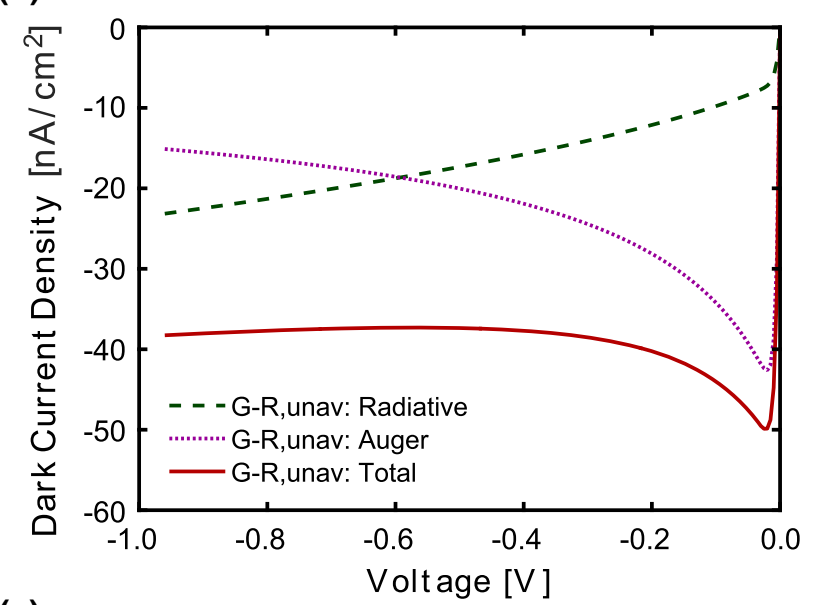

(c)

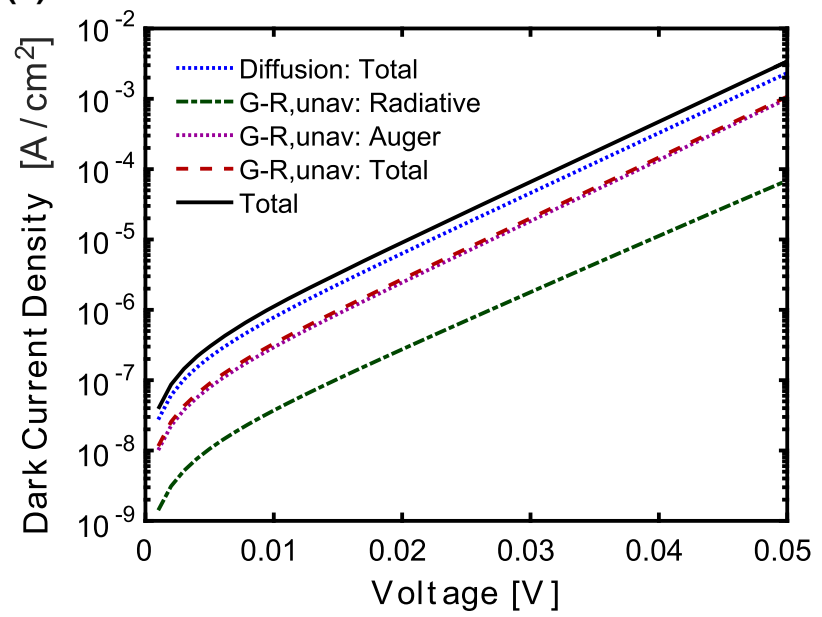

Fig. 3. Analytically modeled LWIR $\mathrm{HgCdTe} P^{+} n^{-}$dark current density versus applied bias for (a) $J_{d-\text { diff. }}, J_{G-R-\text { unav., }}$ and $J_{d-\text { total }}$ $\left(=J_{\text {d-diff. }}+J_{G-R \text {-unav. }}\right)$ under reverse bias, (b) the components of $J_{G-R \text {-unav. }}\left(J_{G-R \text {-unav.rad }}\right.$ and $\left.J_{G-R \text {-unav.-Aug }}\right)$ under reverse bias, and (c) the forward bias current. The temperature is taken to be $60 \mathrm{~K}$ and all other parameters used in the calculation are given in Table I (columns $n^{-}$and $P^{+}$). 


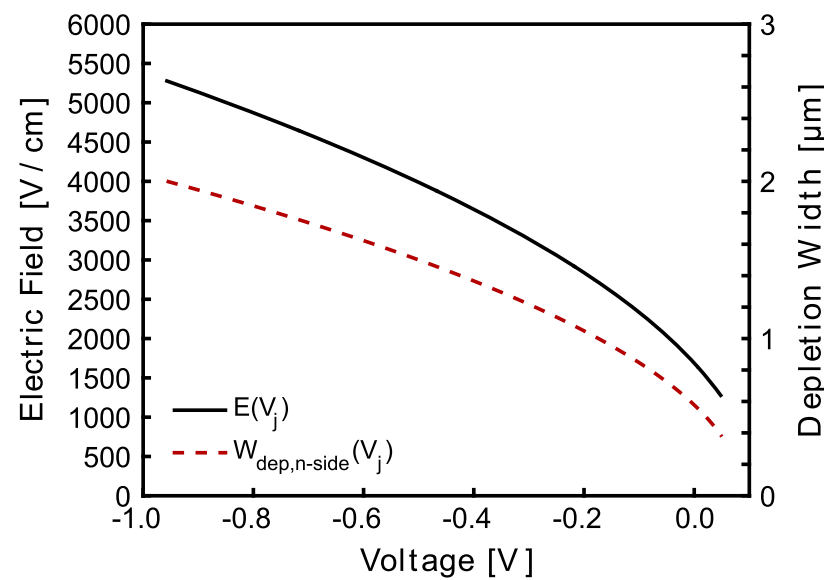

Fig. 4. Analytically modeled LWIR HgCdTe $P^{+} n^{-}$electric field (left $y$-axis) and SCR width (right $y$-axis) versus applied bias. The plotted electric field, calculated using Eq. 2, is $1 / 2$ the maximum electric field. The temperature is taken to be $60 \mathrm{~K}$ and all other parameters used in the calculation are given in Table I (columns $n^{-}$and $P^{+}$).

transitions, which are currently dominant in LWIR detectors and at low temperatures to reduce bandto-band (B2B) tunneling (onset $\sim 40 \mathrm{~K}$ ). In this section, we calculate, using the Evans and Landsberg equations, the dark current density in the $n$ extrinsic region $(T=60 \mathrm{~K})$. This is accomplished by reducing the absorber thickness $d_{n}$ to $2 \mu \mathrm{m}$ and lowering the doping $N_{\mathrm{D}}$, see Table I ( $n^{-}$column). In the "Numerical Validation of Analytical Calculation and Assumptions" section, the results are compared to numerical simulations. The reverse bias dark current characteristics as a function of applied bias for the corresponding $P^{+} n^{-}$structure are plotted in Fig. 3a. As expected, $J_{\mathrm{d} \text {-diff. decreases with reverse }}$ bias and is essentially zero at $V_{j}=-0.967 \mathrm{~V}$. At this voltage, the absorber is fully depleted and the total current $\left(J_{\text {d-total }}\right) \approx J_{G-R \text {-unav. }}$. An interesting observation is that at $V_{j} \sim-0.6 \mathrm{~V}$ the radiative compo-

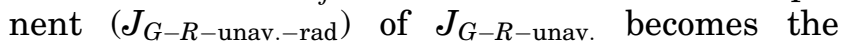
dominant current component; this is illustrated in Fig. 3b. This is understood from Eq. 3, where the Auger term is divided by the electric field. Adequately increasing the reverse bias, such that the electric field is sufficiently large, reduces the Auger term such that the radiative term is dominant.

To illustrate the dependence on the electric field, the electric field versus applied bias is calculated according to Eq. 2 and shown in Fig. 4. The functional dependence of $E\left(V_{j}\right)$ with applied bias is as expected for a one-sided step junction with $N_{\mathrm{D}}<N_{A}^{-}$. Clearly seen is that the field increases linearly with bias. Consequently, given that the field is in the denominator of $J_{G-R \text {-unav.-Aug }}\left(V_{j}\right)$ that current component is eliminated, $\lim _{V_{j} \rightarrow \infty} J_{G-R \text {-unav.-Aug }}$ $\left(V_{j}\right) \rightarrow 0$. An important point is that $J_{G-R \text {-unav.-Aug }}$ is eliminated by the large field, not the large voltage

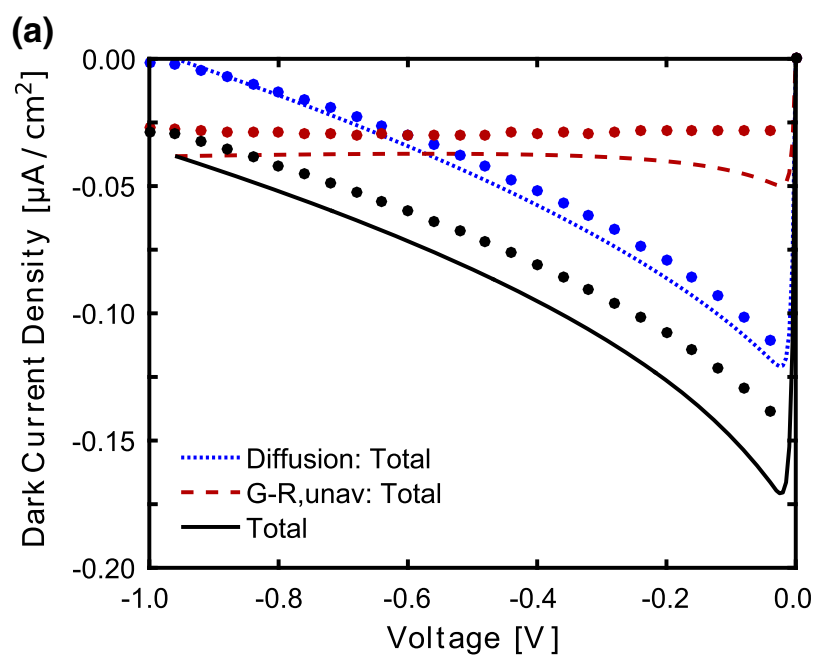

(b)

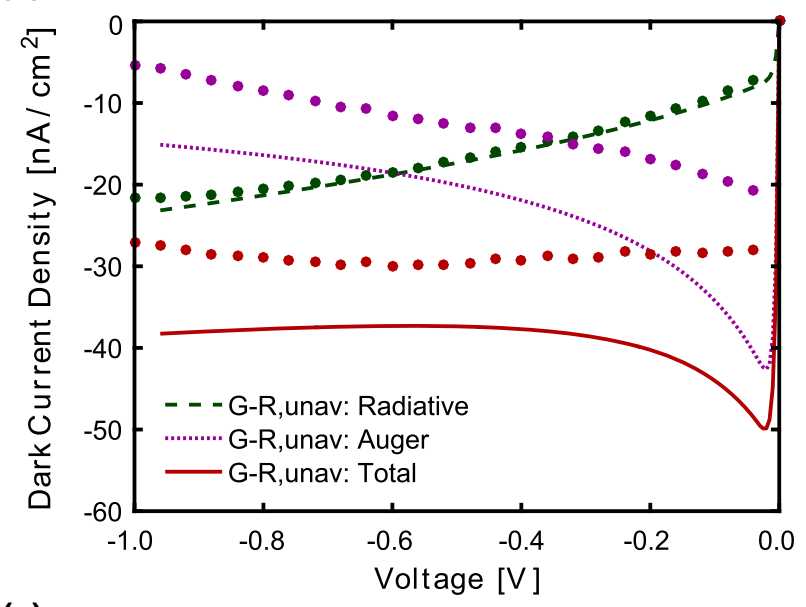

(c)

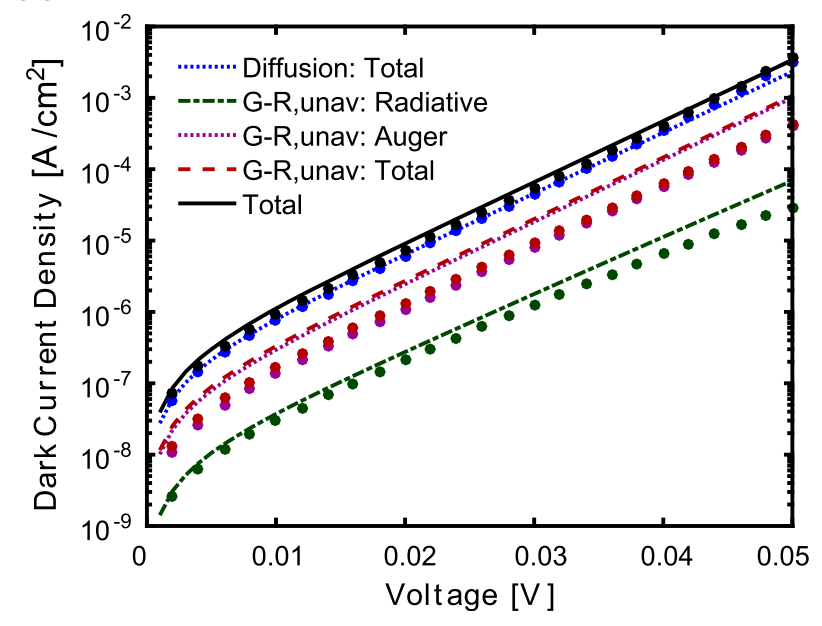

Fig. 5. Comparison of analytical (lines) and numerical (symbols) modeled LWIR HgCdTe $P^{+} n^{-}$dark current density versus applied bias for (a) $J_{d-\text { diff. }}, J_{G-R \text {-unav. }}$, and $J_{d-\text { total }}\left(=J_{d-\text { diff. }}+J_{G-R \text {-unav. }}\right)$ under reverse bias, (b) the components of $J_{G-R \text {-unav. }}\left(J_{G-R \text {-unav-rad }}\right.$ and $J_{G-R \text {-unav.-Aug) }}$ under reverse bias, and (c) the forward bias current. The temperature is taken to be $60 \mathrm{~K}$ and all other parameters used in the calculation are given in Table I (columns $n^{-}$and $P^{+}$). 


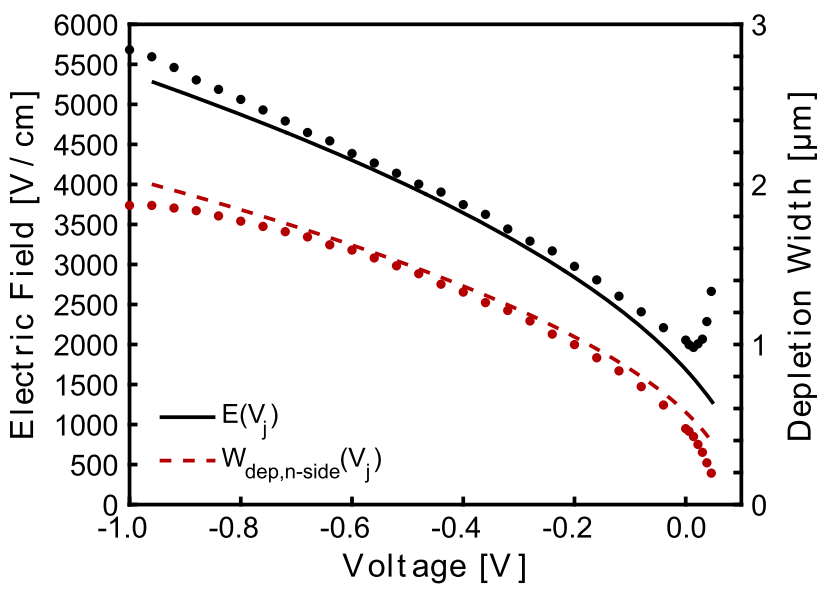

Fig. 6. Comparison of analytical (lines) and numerical (symbols) modeled LWIR HgCdTe $P^{+} n^{-}$electric field (left $y$-axis) and SCR width (right $y$-axis) versus applied bias. The field was calculated analytically using Eq. 2 and extracted numerically by dividing the voltage dependent electrostatic potential by the extracted SCR width as discussed in "Appendix 2". The temperature is taken to be $60 \mathrm{~K}$ and all other parameters used in the calculation are given in Table I (columns $n^{-}$and $P^{+}$).

present, albeit the field is a consequence of the voltage. As such, the same results could be reached at lower voltages, if the field is still sufficiently large.

The trends in the dark current density under forward bias are quite the opposite of those under reverse bias, as is shown in Fig. 3c, calculated using Eqs. 16 and 17. Under forward bias, $W_{\text {dep,n-side }}$ decreases, instead of increasing, and the dominant current is $J_{\mathrm{d} \text {-diff. }}$. Additional contributions from

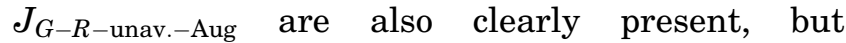
$J_{G-R \text {-unav.-rad }}$ is an order of magnitude lower.

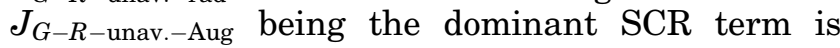
expected, since the Auger term in Eq. 17 is multiplied by an exponential that increases rapidly under forward bias.

\section{NUMERICAL VALIDATION OF ANALYTICAL CALCULATION AND ASSUMPTIONS}

An objective of this paper is to validate the analytical generalized Evans and Landsberg current density equations using numerical simulations. With the assumptions that were made in deriving the analytical equations, such as the use of the depletion approximation and the mass-action law $\left(n_{n 0} p_{n 0}=n_{\mathrm{i}}^{2}\right)$, it is clearly instructive to do so. In order to best replicate the analytical calculation, two-dimensional (2D) numerical simulations were performed of an idealized device without any lateral variation. For the details of the numerical simulations, see "Appendix 2". Figure 5a presents a comparison of $J_{\mathrm{d} \text {-diff., }} J_{G-R \text {-unav., and } J_{\mathrm{d} \text {-total }}}$ $\left(=J_{\text {d-diff. }}+J_{G-R \text {-unav. }}\right)$ for both the analytical calculation (lines) and numerical simulation (symbols) under reverse bias. Clearly seen is that both models (a)

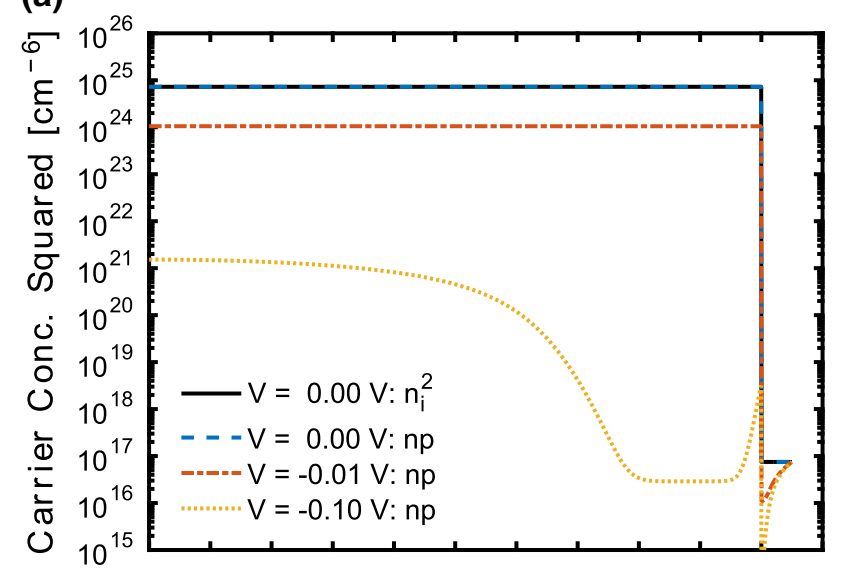

(b)

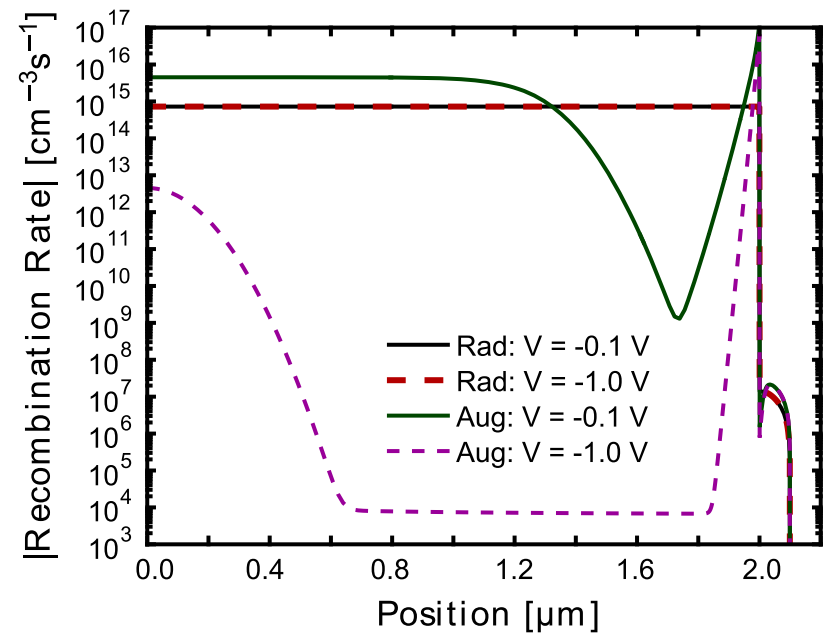

Fig. 7. Numerically modeled carrier concentration squared (a), and recombination rates (b) for the LWIR HgCdTe $P^{+} n^{-}$hetero-structure diode at $T=60 \mathrm{~K}$ at various bias values.

predict the same identical trends and that $J_{\mathrm{d}-\text { total }}$ is very similar between the two. $J_{\mathrm{d} \text {-diff. is slightly }}$ smaller in the numerical simulations due to fewer Auger transitions which alters the $J_{G-R-\text { unav. }} /$ $J_{\text {d-diff. crossover voltage. Most importantly, both }}$ models predict that $J_{G-R-\text { unav. }}$ emerges as the sole source of dark current as $V_{j} \rightarrow-1.0 \mathrm{~V}$, which corresponds to when the absorber is fully depleted.

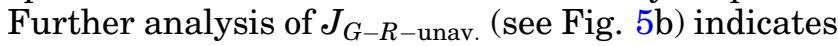
that the models deviate at larger reverse bias voltage with the analytical calculation yielding a slightly larger Auger contribution $\left(J_{G-R \text {-unav.-Aug }}\right)$. However, both models yield very similar values for $J_{G-R-\text { unav.-rad }}$ and predict the transition of the dominant current mechanism from Auger to radiative at $\sim-0.6 \mathrm{~V}$, confirming that the LWIR diode is in fact radiatively limited. This key prediction from the analytical calculation has now been numerically verified. Plotted in Fig. $5 \mathrm{c}$ is the forward bias current for both models. The agreement between the two is excellent. In both models, the dominant current contribution is $J_{\mathrm{d} \text {-diff. followed by }}$ 


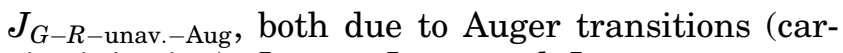
rier injection). $J_{\mathrm{d} \text {-total }}, J_{\mathrm{d} \text {-diff. }}$ and $J_{G-R \text {-unav.-rad }}$ are very similar between both calculations, however, $J_{G-R \text {-unav.-Aug is smaller in the numerical }}$ simulation.

Evident in Fig. 5a, b, and c is some discrepancy between the results from the two models, despite the fact that the same key conclusions were reached in each. Under large reverse bias, the numerical simulation predicts fewer Auger transitions than the analytical calculation, therefore smaller $J_{\mathrm{d} \text {-diff. }}$

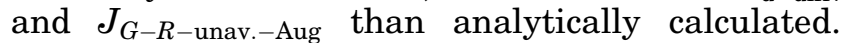
This difference is not likely due to the electric field, as it is very similar in both models (see Fig. 6), albeit slightly larger in the numerical simulations. Most importantly, in the numerical simulation charge neutrality is broken. Specifically, shown in Fig. $7 \mathrm{a}$ is $n p=n_{\mathrm{i}}^{2}$ at $V_{j}=0 \mathrm{~V}$, but $n p \ll n_{\mathrm{i}}^{2}$ at $V_{j}<0 \mathrm{~V}$. This contradicts the key assumption of the analytical calculation $\left(n p=n_{\mathrm{i}}^{2}\right)$, and thus is a probable source of the difference in values between the two models. Interestingly, the Auger recombination rate changes by many orders of magnitude, due to its squared dependence on the carrier concentrations, but the radiative recombination rate changes negligibly with reverse bias (see Fig. $7 b$ ). Therefore, the radiative recombination rate is nearly invariant to the carrier concentration, which is why, according to Eq. 6, it is expected to be the sole recombination mechanism contributing to the dark current in fully depleted devices when the carrier concentrations have been significantly reduced. Additional possibilities for the differences between the two models extend from the realization that in the numerical simulation, the SCR region extends completely to the back surface of the device, and the recombination rates continually decrease with larger voltages.

\section{INTRINSIC REGION}

The Evans and Landsberg theory is not expected to be valid in the intrinsic region of operation, where $n_{\mathrm{i}} \gg N_{\mathrm{d}}$ and $n_{n 0} \sim p_{n 0} \sim n_{\mathrm{i}}$. As such, the numerical model is extended into the intrinsic region by increasing the temperature. This region is especially important due to the push to operate IR detectors at higher temperatures. ${ }^{4}$ Figure 8 presents the numerically modeled dark current density versus applied bias for the DLPH $P^{+} n^{-}$heterostructure diode at (a) $T=60 \mathrm{~K}$, (b) $T=125 \mathrm{~K}$ and, (c) $T=200 \mathrm{~K}$. Increasing the temperature beyond $125 \mathrm{~K}$, places the diode in the intrinsic region where $n_{\mathrm{i}} \gg N_{\mathrm{D}}$ in the AL. Note that the large spike at small biases in $J_{\text {d-diff. at }} T=200 \mathrm{~K}$ is due to the width of the SCR decreasing as the temperature is increased. As such, a larger bias is required to appreciably enlarge the SCR to reduce $J_{\mathrm{d} \text {-diff. }}$. Fortunately, at all temperatures the same trends are observed, $J_{\mathrm{d} \text {-diff. }} \rightarrow 0$ at large reverse bias and $J_{G-R-\text { unav. }}$ surpassed $J_{\mathrm{d} \text {-diff. }}$ as the dominant
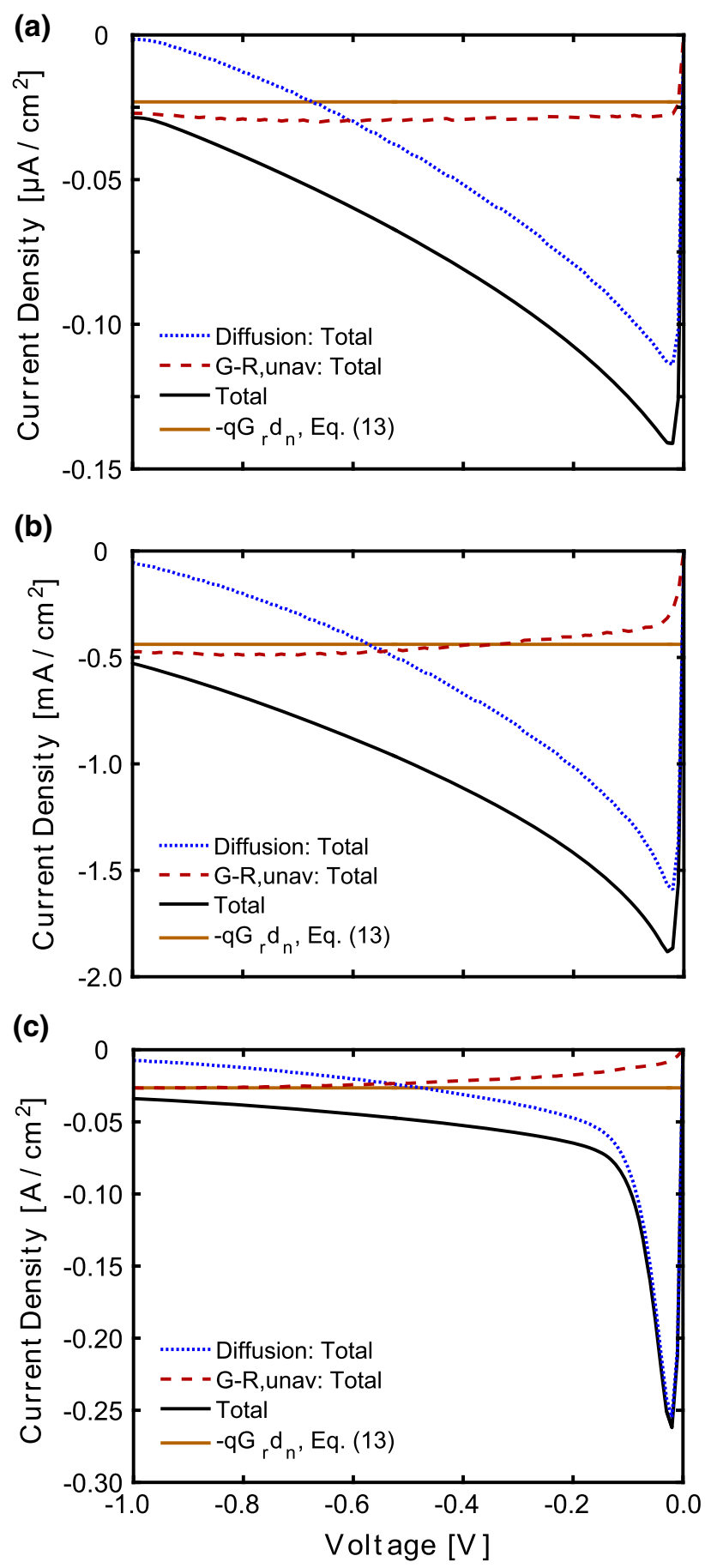

Fig. 8. Numerically simulated LWIR HgCdTe $P^{+} n^{-}$dark current density versus applied bias at (a) $T=60 \mathrm{~K}\left(N_{\mathrm{d}} \gg n_{\mathrm{i}}\right)$, (b) $T=125 \mathrm{~K}$ $\left(n_{\mathrm{i}} \sim N_{\mathrm{d}}\right)$, and $(\mathrm{c}) T=200 \mathrm{~K}\left(n_{\mathrm{i}} \gg N_{\mathrm{d}}\right)$.

current mechanism, with radiative recombination being the dominant contribution to $J_{G-R \text {-unav. at }}$ large reverse bias. These same trends being observed in the intrinsic region, while perhaps not surprising, are nonetheless important to verify given the extreme interest in HOT detectors.

Furthermore, it was theorized in Eq. 6 that for a fully depleted device, $J_{\text {d-diff. }} \rightarrow 0$ because 
$d_{n}-W_{\text {dep,n-side }}\left(V_{j}\right)=0$ and $J_{G-R \text {-unav.-Aug }} \rightarrow 0$ due to the large field, and the dark current would be given by $J_{\text {Fully-Depleted }}=-q G_{R} d_{n}$. The radiative recombination rate $G_{R}$ was shown by Blakemore ${ }^{12}$ to depend on the absorption coefficient through the following equation

$$
G_{R}=\frac{8 \pi}{h^{3} c^{2}} \int_{0}^{\infty} \frac{\epsilon\left(E_{\mathrm{ph}}\right) \alpha\left(E_{\mathrm{ph}}\right) E_{\mathrm{ph}}^{2} d E_{\mathrm{ph}}}{\exp \left[E_{\mathrm{ph}} /\left(k_{B} T\right)\right]-1}
$$

where $h$ is Planck's constant, $c$ is the speed of light, $E_{\mathrm{ph}}$ is the photon energy, and $\epsilon\left(E_{\mathrm{ph}}\right)$ is the relative dielectric constant. The values for $G_{R}$ that Eq. 12 produce depend heavily on the optical model used, through both the parameters $\epsilon\left(E_{\mathrm{ph}}\right)$ and $\alpha\left(E_{\mathrm{ph}}\right)$. For example, if $\alpha\left(E_{\mathrm{ph}}\right)$ is calculated assuming parabolic bands, evaluating Eq. 12 yields the following expression for the radiative recombination rate ${ }^{15}$ :

$$
\begin{aligned}
G_{R} & =5.8 \times 10^{-13} \epsilon_{\infty}^{1 / 2} n_{\mathrm{i}}^{2} \\
& \times\left(\frac{m_{0}}{m_{e}^{*}+m_{h}^{*}}\right)^{3 / 2}\left(1+\frac{m_{0}}{m_{e}^{*}}+\frac{m_{0}}{m_{h}^{*}}\right)\left(\frac{300}{T}\right)^{3 / 2} \\
& \times\left(E_{g}^{2}+3 k_{B} T E_{g}+3.75 k_{B}^{2} T^{2}\right),
\end{aligned}
$$

which is the expression used throughout this analysis (values given in Table I). The details of which are given in Ref. 15, eqs. $(4,5)$. Equation 13 is commonly used in $\mathrm{HgCdTe}$ device analysis, and used here in comparing the analytical and numerical calculations. $J_{\text {Fully-Depleted }}$ is calculated using Eq. 13 and is plotted in Fig. 8 as the tan lines. The lines are invariant to applied bias because $d_{n}$ has been used in the calculation, which is a constant, instead of $W_{\text {dep,n-side }}\left(V_{j}\right)$. Clearly, the numerical simulations converge to this asymptotic value. This is expected since the value for the radiative recombination coefficient $B_{0}$ input into the numerical model was calculating from Eq. 13 according to $B_{0}=G_{R} / n_{\mathrm{i}}^{2}$. Additionally, since the numerical simulations converge to $J_{\text {Fully-Depleted }}$, calculated using Eq. 6, the initial analytical prediction that a fully depleted diode is radiatively limited has now been numerically verified.

Stated previously was that the values of $G_{R}$ calculated from Eq. 12 depend heavily on the optical model used. Consider now the calculation of $G_{R}$ from Eq. 12 using the empirically derived Schacham and Finkman absorption model ${ }^{15}$ whose value of $\alpha\left(E_{\mathrm{ph}}\right)$ agree far better with experimental measurements than the previous parabolic band approximation. Evaluating Eq. 12 with the Schacham and Finkman absorption model yields the following expression for the radiative recombination rate $^{15}$ :

$$
\begin{aligned}
G_{R} & =2.8 \times 10^{17} \epsilon_{\infty} T^{3 / 2} \exp \left(\frac{-E_{g}}{k_{B} T}\right) \\
& \times 2.109 \times 10^{5}\left(\frac{1+x}{81.9+T}\right)^{1 / 2} \\
& \times\left(E_{g}^{2}+3 k_{B} T E_{g}+3.75 k_{B}^{2} T^{2}\right) .
\end{aligned}
$$

The details of which are given in Ref. 15, eqs. (610). Calculating $J_{\text {Fully-Depleted }}$ using Eq. 14 yields slightly larger values for the dark current than Eq. 13, especially at lower temperature. Since Eq. 14 yields larger values than Eq. 13 for $G_{R}$, due to the absorption coefficient being larger in magnitude and having a longer Urbach tail, the result of using Eq. 14 is to decrease the radiative minority carrier lifetime, since

$$
\tau_{\mathrm{rad}}=\frac{n_{\mathrm{i}}^{2}}{G_{R}\left(n_{0}+p_{0}\right)} .
$$

and to noticeably increase the dark current density, as shown in Fig. 8. This corresponds to the radiative lifetime being shorter by a factor of 2.3 at $60 \mathrm{~K}$ and 1.7 at $125 \mathrm{~K}$. The difference in values in $G_{R}$ associated with using Eq. 13 versus Eq. 14 has long been known. ${ }^{15}$

\section{CONCLUSIONS}

Under the condition that intrinsic recombination mechanisms (Auger and radiative) are the dominant current components, in the situation that the SCR occupies a significant volume of the absorbing layer, the Evans and Landsberg paper ${ }^{1}$ is of the utmost importance in understanding the physics of $p-n$ junctions. In this paper, we have utilized the theory of Evans and Landsberg, which states current generated in the SCR depends on the intrinsic radiative and Auger coefficients, and extended that theory to large reverse bias. The Evans and Landsberg current density equations were validated in the $n$-extrinsic region $\left(N_{\mathrm{d}} \gg n_{\mathrm{i}}\right)$ with analytical and numerical simulations and we observed that, while the Auger recombination rate changes drastically with carrier concentration (applied reverse bias), the radiative recombination rate remains nearly constant with applied reverse bias. Subsequently, the analysis was numerically extended to the intrinsic region $\left(n_{\mathrm{i}} \gg N_{\mathrm{d}}\right)$, especially relevant given the drive towards HOT detectors. It was shown, in both regions, that at sufficiently large reverse bias, such that absorber is fully depleted, the dark current is given by $J_{\text {Fully-Depleted }}=-q G_{R} d_{n}$, where $d_{n}$ is the absorber thickness and $G_{R}$ is the radiative recombination rate, calculated by integrating the measured optical absorption coefficient $\alpha\left(E_{\mathrm{ph}}, T\right)$ over all photon energies using the theory of van Roosbroeck and Shockley. ${ }^{2}$ This equation represents the fundamental performance limit for a detector and is attributed to (1) the QNR vanishing, which eliminates the diffusion current, and (2) the increasing electric field (with increasing applied reverse bias), which suppresses Auger transitions in the SCR. Therefore, the dark current in optimized depleted devices is given by the intrinsic radiative process, enabling radiatively limited detectors which will elevate the performance of existing systems by contributing less thermal noise at 
existing temperatures or enabling equivalent performance at higher temperatures.

\section{ACKNOWLEDGEMENT}

The simulations were conducted using computer cores and Synopsys TCAD Sentaurus licenses located within the Sensors and Electron Devices Directorate (SEDD) at the Army Research Laboratory (ARL).

\section{APPENDIX 1: EVANS AND LANDSBERG CURRENT DENSITY EQUATIONS}

In the seminal paper by Evans and Landsberg ${ }^{1}$ on recombination statistics in $p-n$ junctions, a generalization is presented of the usual SRH recombination statistics to include the effect of radiative and Auger transitions in the SCR. This yielded a revised expression for the steady-state recombination rate of electrons and holes. This was accomplished by considering all of the allowable transitions in a semiconductor sample and writing expressions for the recombination rates. This exercise identified a number of recombination processes, including socalled unavoidable processes (unav.). Integrating the unavoidable recombination rate over the $p-n$ junction SCR yields the dark current due to unavoidable recombination $\left(J_{G-R-\text { unav. }}\right) . J_{G-R \text {-unav. }}$ is unique from the usual $G-R$ rate that occurs in the SCR of a $p-n$ junction due to transitions between defect levels in the bandgap $\left(J_{G-R-S R H .}\right)$. The equations for the dark current generated in the QNR due to diffusion $\left(J_{\mathrm{d} \text {-diff. }}\right)$ and unavoidable recombination in the $\operatorname{SCR}\left(J_{G-R \text {-unav. }}\right)$ of a $P^{+} n$ hetero-structure photodiode (assuming the only contributions are from the $n$-side and not the $P^{+}$. side), is given by ${ }^{1}$

$$
\begin{aligned}
& \begin{aligned}
J_{\mathrm{d}-\text { diff. }}\left(V_{j}, T\right)=q n_{\mathrm{i}}^{2} & \left\{\left(B_{0}+n_{n 0} B_{1}\right)\left[d_{n}-W_{\text {dep }, \mathrm{n}-\text { side }}\left(V_{j}\right)\right]\right\} \\
\times\left[\exp \left(\beta_{T} V_{j}\right)-1\right] & \\
J_{G-R-\text { unav. }}\left(V_{j}, T\right) & =q n_{\mathrm{i}}^{2}\left\{B_{0}+\left(n_{n 0} B_{1}+p_{p 0} B_{2}\right)\right. \\
& \left.\times\left[\frac{1-\exp \left[-2\left(\eta_{\mathrm{d}}-\eta_{j}\right)\right]}{2\left(\eta_{\mathrm{d}}-\eta_{j}\right)}\right]\right\} \\
& \times W_{\text {dep }, \mathrm{n}-\text { side }}\left(V_{j}\right)\left[\exp \left(\beta_{T} V_{j}\right)-1\right],
\end{aligned}
\end{aligned}
$$

where,

$$
\eta_{\mathrm{d}}=\frac{V_{b i}}{2 k_{B} T}, \quad \eta_{j}=\frac{V_{j}}{2 k_{B} T}
$$

An important observation is the Auger (h-h) tran-

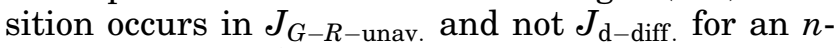
type one-sided $P^{+} n$ hetero-structure photodiode.

The total reverse bias dark current $\left(V_{j}<-2 / \beta_{T}\right)$ is given by

$$
\begin{gathered}
J_{\mathrm{d}-\text { total }}\left(V_{j}, T\right)=-q n_{\mathrm{i}}^{2}\left\{\left(B_{0}+n_{n 0} B_{1}\right)\left[d_{n}-W_{\text {dep }, \mathrm{n}-\text { side }}\left(V_{j}\right)\right]\right. \\
\left.+B_{0} W_{\text {dep }, \mathrm{n}-\text { side }}\left(V_{j}\right)+k_{B} T\left(\frac{n_{n 0} B_{1}+p_{p 0} B_{2}}{E\left(V_{j}\right)}\right)\right\}
\end{gathered}
$$

where

$$
E\left(V_{j}\right)=\frac{V_{b i}-V_{j}}{W_{\text {dep, }, \text {-side }}\left(V_{j}\right)}
$$

and $E\left(V_{j}\right)$ is $1 / 2$ the maximum electric field of an abrupt one-sided step junction in the depletion approximation, with $n_{n 0}<p_{p 0}$.

\section{APPENDIX 2: NUMERICAL SIMULATION}

The authors have long used a three-dimensional (3D) finite element method implementation to solve Poisson's equation and the continuity equations. ${ }^{16}$ The numerical simulation is discussed in detail in Ref. 17 where it has been used to simulate pixel arrays. Specifically, for simulations of pixel arrays, the 3D numerical simulation uses at minimum a $3 \times 3$ or mini-array with the center pixel as the "pixel diode" under test. All diodes are uniformly biased so the "pixel diode" characteristic emulates the diode in a realistic dense array. ${ }^{3}$ The model can also accommodate larger arrays at any pixel pitch or bias configuration completely capturing dense array effects. ${ }^{18}$ Since the focus of this work is on studying effects along the growth direction (vertical QNR and SCR currents) and not lateral/3D effects, the simulations in this manuscript were conducted using a $2 \mathrm{D}$ rather than a 3D numerical simulation. Reducing the dimensionality from $3 \mathrm{D}$ to $2 \mathrm{D}$ immensely reduces the computational complexity, making the process of mapping the parameter space of pertinent sensitivity parameters far more tractable. The material parameters used in the numerical simulation are identical to those used in the analytical calculation and given in Table I.

As stated previously a 2D finite element method implementation is used to solve Poisson's equation and the continuity equations. The model outputs: (1) the total current at each contact and (2) a 2D/3D computer-aided design file with graphical representation of the device structure (and numerical mesh), with semiconductor parameters, carrier densities, recombination rates, etc. at each mesh point. The goal of this manuscript is to compare the numerical simulation results to the analytically calculated

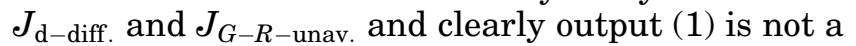

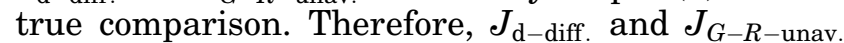
must be extracted from output (2). Given that output (2) is a $2 \mathrm{D} / 3 \mathrm{D}$ file, line-cuts are performed to yield the radiative and Auger recombination rates versus device thickness along the epitaxial direction. An exemplar of each versus device 


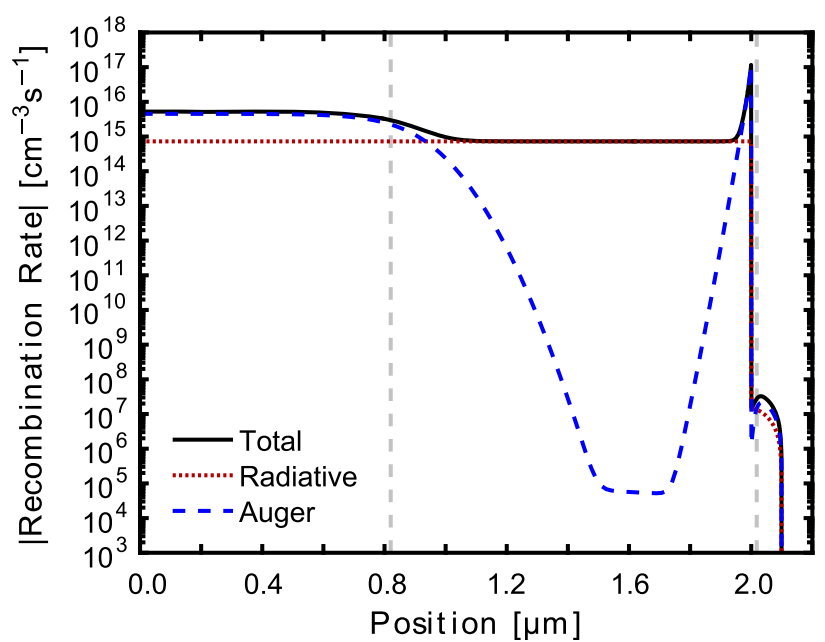

Fig. 9. Example of the numerically modeled recombination rates versus device thickness at an applied bias of $V_{j}=-0.3 \mathrm{~V}$. The metallurgical junction resides at $z=2.0 \mu \mathrm{m}$ and the gray lines denote the edge of the SCR.

thickness are shown in Fig. 9. Note that at this point the current is divided by recombination mechanism, but not by where it was generated, such as inside or outside the SCR (i.e. $J_{\text {d-diff. versus }}$ $\left.J_{G-R \text {-unav. }}\right)$. The goal of the current work is to separate SCR currents $\left(J_{G-R \text {-unav. }}\right)$ from QNR diffusion currents $\left(J_{\mathrm{d} \text {-diff. }}\right)$, a simple task if the depletion approximation is used. Since the numerical simulation does not use the depletion approximation, it is impossible for it to directly output $J_{\mathrm{d} \text {-diff. }}$ and $J_{G-R \text {-unav. }}$ As such, the recombination rates that the model does output must be manipulated to separate out the contributions that would coincide to the SCR and QNR, if the depletion approximation had been used. Using the electrostatic potential, the recombination rates can be partitioned into the SCR contribution and QNR contribution. In the depletion approximation, the electrostatic potential switches from its equilibrium value in the $p$ and $n$ layers respectively, in an amount equal to the built in voltage, and the region that the potential changes over corresponds to the SCR. The SCR was taken to reside where the electrostatic potential deviates from its equilibrium value by $1 \%$ (in the depletion approximation the SCR largely coincides with where the potential changes ${ }^{19}$ ). While this approach consistently yielded the expected results for the $n$ side of the SCR (increasing with reverse bias and nearly identical to the analytically calculated value), the $p$-side of the SCR incorrectly decreased with increasing reverse bias. An alternate approach is to instead define the SCR as where the carrier concentrations decrease from their equilibrium values by $50 \%$. Following this approach, the SCR on both the $n$ - and $p$-sides increases with increasing reverse bias and the $n$-side results are nearly identical to the electrostatic potential approach. Using the aforementioned approach, the edges of the SCR are shown in gray in Fig. 9. The recombination rates are then split into the constituent contributions that correspond to outside and inside the arbitrarily defined SCR, then subsequently integrated to yield $J_{\mathrm{d} \text {-diff. }}$ and $J_{G-R \text {-unav. }}$ respectively. Specifically:

$$
\begin{gathered}
J_{\mathrm{d}-\text { diff. }}=\int_{\mathrm{QNR}} U_{\mathrm{rad}}(z) d z+\int_{\mathrm{QNR}} U_{\mathrm{Aug}}(z) d z \\
J_{G-R \text {-unav. }}=\int_{\mathrm{SCR}} U_{\mathrm{rad}}(z) d z+\int_{\mathrm{SCR}} U_{\mathrm{Aug}}(z) d z
\end{gathered}
$$

where $U(z)$ is the recombination rate and $z$ is the epitaxial direction.

\section{REFERENCES}

1. D. Evans and P. Landsberg, Solid-State Electron. 6, 169 (1963).

2. W. Van Roosbroeck and W. Shockley, Phys. Rev. 94, 1558 (1954).

3. R. DeWames, R. Littleton, K. White, A. Wichman, E. Bellotti, and J. Pellegrino, J. Electron. Mater. 44, 2813 (2015).

4. D. Lee, M. Carmody, E. Piquette, P. Dreiske, A. Chen, A. Yulius, D. Edwall, S. Bhargava, M. Zandian, and W.E. Tennant, J. Electron. Mater. 45, 4587 (2016).

5. R. DeWames, P. Maloney, C. Billman, and J. Pellegrino, in Infrared Technology and Applications XXXVII, Proceedings of SPIE, vol. 8012:801239 (2011).

6. M.A. Kinch, State-of-the-Art Infrared Detector Technology (SPIE Press, Bellingham, 2014).

7. J. Bajaj, J.M. Arias, M. Zandian, D.D. Edwall, J.G. Pasko, L.O. Bubulac, and L.J. Kozlowski, J. Electron. Mater. 25, 1394 (1996).

8. J.M. Arias, J.G. Pasko, M. Zandian, J. Bajaj, L.J. Kozlowski, R.E. DeWames, and W.E. Tennant, in Producibility of II-VI Materials and Devices, Proceedings of SPIE, vol. 2228, pp. 210-224 (1994).

9. C.A. Grimbergen, Solid-State Electron. 19, 1033 (1976).

10. J. Schuster, R.E. DeWames, E.A. DeCuir Jr., E. Bellotti, and P.S. Wijewarnasuriya, Appl. Phys. Lett. 107, 023502 (2015).

11. J. Schuster, R.E. DeWames, E.A. DeCuir Jr., E. Bellotti, N. Dhar, and P.S. Wijewarnasuriya, in Infrared Sensors, Devices, and Applications V, Proceedings of SPIE, vol. 9609:960904 (2015).

12. J.S. Blakemore, Semiconductor Statistics (Pergamon Press, Oxford, 1962).

13. W.E. Tennant, J. Electron. Mater. 39, 1030 (2010).

14. G.L. Hansen, J.L. Schmit, and T.N. Casselman, J. Appl. Phys. 53, 7099 (1982).

15. S.E. Schacham and E. Finkman, J. Appl. Phys. 57, 2001 (1985).

16. Synopsys, Sentaurus Device User Guide (2015), version K2015.06.

17. J. Schuster, B. Pinkie, S. Tobin, C. Keasler, D. D'Orsogna, and E. Bellotti, IEEE J. Sel. Top. Quantum Electron. 19, 3800415 (2013)

18. A.R. Wichman, R.E. DeWames, and E. Bellotti, in Infrared Technology and Applications XL, Proceedings of SPIE, vol. 9070:907003 (2014).

19. W. Shockley, Bell Lab. Tech. J. 48, 435 (1949). 